\title{
PILEFLEBITIS POSTERIOR A APENDICITIS AGUDA REPORTE
} DE CASO

\author{
Pylephlebitis after acute appendicitis, case report.
}

Christian García Quirós1 1 Jairo Alfaro Alfaro²

1, Especialista de Medicina Interna Hospital San Juan de Dios, Caja costarricense de seguro social, San José, Costa Rica. 2, Médico general, Trabajador independiente, San José, Costa Rica.

\section{RESUMEN}

La pileflebitis o piletromboflebitis corresponde a la trombosis séptica de la vena porta y de sus ramas debido a un foco infeccioso a nivel intraabdominal. Entre las etiologías más frecuentes podemos encontrar la diverticulitis, la apendicitis y colangitis. También se puede asociar a estados de hipercoagulabilidad, neoplasias y deficiencia de factores de la coagulación.

El tratamiento se enfoca en la erradiación del foco infeccioso mediante terapia antibiótica y en la prevención de su extensión e isquemia mesentérica mediante la anticoagulación.

Describimos el caso de un paciente con antecedente de apendicitis aguda, quien

\section{Cómo citar:} desarrolla trombosis séptica de la vena porta, esplénica y mesentérica superior.

García Quirós, C., \& Alfaro Alfaro, J. (2021). PILEFLEBITIS POSTERIOR A APENDICITIS AGUDA. Revista Ciencia Y Salud, 5(2), Pág. 40-45.

Palabras Clave: Pileflebitis; tromboflebitis séptica; apendicitis; trombosis portal; trombosis esplenomesentérica.

Recibido: 16/ene/2021

Aceptado: 01/mar/2021

Publicado: 16/abr/2021

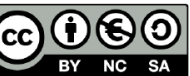




\section{CIENCIA\&SALUD}

\section{ABSTRACT}

Pylephlebitis refers to the septic thrombosis of the portal vein and its branches due to an infectious focus at the intra-abdominal level. Among the most frequent etiologies we can find diverticulitis, appendicitis and cholangitis. It can also be associated with hypercoagulable states, neoplasms and deficiency of coagulation factors.

The treatment focuses on the eradication of the infectious focus through antibiotic therapy and in the prevention of its extension and mesenteric ischemia through anticoagulation.

We describe the case of a patient with a history of acute appendicitis that develops septic thrombosis of the portal vein, splenic vein and superior mesenteric vein.

Keywords: Pileflebitis; septic thrombophlebitis; appendicitis; portal vein thrombosis; Spleno-mesenteric thrombosis

\section{Caso Clínico}

Masculino de 34 años, costarricense, vecino de La Carpio, San José. Desconocido enfermo, sin alergia a medicamentos, tabaquismo ni toxicomanías. Con antecedente de apendicectomía no complicada el 19 de octubre del 2018. Consulta al servicio de emergencias de un Hospital en el postoperatorio \#22 días, por dolor abdominal de predominio en epigastrio e hipocondrio derecho, vómitos y sensación febril desde su egreso hospitalario. Fue valorado inicialmente en el servicio de emergencias quirúrgicas donde se realiza ultrasonido abdominal que evidencia trombosis de la vena mesentérica superior, confluencia esplenoportal y porta. Los estudios de laboratorio revelaron recuento de glóbulos blancos $11240 / \mathrm{mm} 3$, fosfatasa alcalina $403 \mathrm{U} / \mathrm{I}$, gamma glutamil transpeptidasa $404 \mathrm{UI} / \mathrm{I}$ y amilasa $28 \mathrm{U} / \mathrm{L}$.

Al examen físico se describe eupnéico, hidratado, febril, pálido con fascies álgica. Cicatriz de apendicectomía con adecuada cicatrización, sin eritema periférico, dehiscencias o secreción, abdomen blando, depresible, levemente doloroso a la palpación de epigastrio con cierta defensa muscular. Sin datos de irritación peritoneal.

Se indica anticoagulación con warfarina $5 \mathrm{mg}$ al día y enoxaparina $60 \mathrm{mg}$ cada 12 horas SC y seguimiento en la consulta externa de Vascular periférico en 10 días.

El paciente persiste con picos febriles de 39ㄷ diariamente, con dolor abdominal y con mal estado general. Se presenta a la consulta de vascular periférico a los 10 días donde se le documenta INR en 5.85, se indica modificar dosis de warfarina a $2.5 \mathrm{mg}$ por día y se refiere al servicio de emergencias para su valoración. En el servicio de emergencias médicas se encuentra con $39^{\circ} \mathrm{C}$, frecuencia cardíaca en 74 lpm, presión arterial en 158/108 mmHg. Se describe álgico, con abdomen blando, depresible, levemente distendido, sin déficit neurológico. 


\section{CIENCIA\&SALUD}

Se solicitan hemocultivos y laboratorios control que reportan glóbulos blancos $11860 / \mathrm{mm} 3$ con predominio de $66 \%$ de polimorfonucleares, sin bandemia, fosfatasa alcalina $250 \mathrm{U} / \mathrm{I}$, gamma glutamil transpeptidasa 310 UI/I, amilasa $31 \mathrm{U} / \mathrm{L}$, sin alteraciones en la función renal, electrolitos ni elevación de bilirrubinas. Los hemocultivos resultaron negativos a los 7 días.

Se inicia terapia antibiótica empírica con cefotaxime y metronizadol y se solicita TAC de abdomen con medio de contraste el cual documenta trombosis porto-espleno-mesentérica con escaso líquido libre en fosa iliaca derecha, sin evidencia de colecciones intraabdominales.

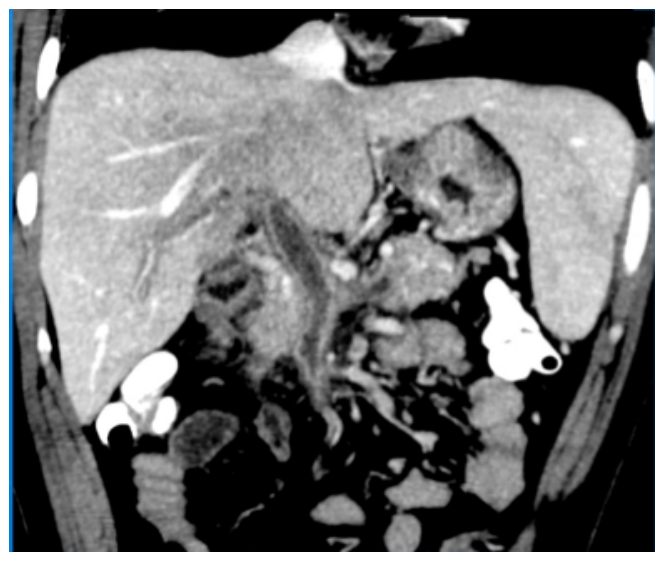

Figura 1. Tomografía axial computerizada con contraste intravenoso, muestra la trombosis en la vena porta.

Se decide su ingreso hospitalario para continuar terapia antibiótica y optimizar dosis de anticoagulación. Al ingreso se documenta PCR en $173.5 \mathrm{mg} / \mathrm{L}$ y procalcitonina en $2.7 \mathrm{ng} / \mathrm{mL}$.

Completó cobertura con cefotaxime $1 \mathrm{~g}$ IV cada 4 horas y metronidazol 500 mg cada 8 horas por 14 días. A su vez, se ajusta dosis de warfarina hasta alcanzar dosis óptimas de anticoagulación titulando diariamente según INR.

La evolución clínica durante su estancia hospitalaria fue satisfactoria, con desaparición de la fiebre a los tres días y descenso de reactantes de fase aguda.

Durante su internamiento se envía perfil por trombofilias para ser valorado en la consulta externa.

Paciente se egresa con ciprofloxacina oral para completar 4 semanas de terapia antibiótica.

\section{Discusión y análisis del caso}

La endoflebitis supurativa de la vena porta (pileflebitis), como complicación de la apendicitis aguda, fue descrita por primera vez por Waller en 1846 (1).

La pileflebitis es una complicación rara de un proceso inflamatorio intraabdominal o de cualquier foco intraperitoneal cuyo drenaje venoso dependa del sistema portal.

En un estudio retrospectivo que evaluó a 7796 pacientes con infecciones intraabdominales en un Hospital de tercer nivel durante 10 años, se demostró una incidencia de pileflebitis de 0.37 casos por 100000 habitantes/año (2). 


\section{CIENCIA\&SALUD}

Esta entidad se puede presentar a cualquier edad, desde neonatos hasta adultos (3).

El número de casos que se han reportado durante la última década equivale al número reportado en las últimas tres décadas, por lo que es claro que con el uso del ultrasonido abdominal y la tomografía axial computarizada se ha facilitado la detección temprana de esta entidad y la prevención de las complicaciones asociadas (3).

En una revisión de 100 reportes de casos de pacientes con pileflebitis, Kanellopoulou, et. al. concluyen que la causa más frecuente en el 30\% de los casos corresponde a diverticulitis, seguida de apendicitis en el $19 \%$ y enfermedad inflamatoria intestinal en el 6\%(3).

Antes se consideraba la apendicitis como la etiología más común, sin embargo el manejo quirúrgico temprano en la apendicitis aguda y el manejo conservador en la diverticulitis pueden influir en el aumento en la frecuencia de estas otras etiologías.

La presencia de factores que predisponen a estados de hipercoagulabilidad puede facilitar el desarrollo de tromboflebitis séptica. Entre estos factores, asociados se han descrito: malignidad (principalmente enfermedades mieloproliferativas), $\mathrm{VIH}$, deficiencia de antitrombina III, presencia de anticoagulante lúpico y la deficiencia de proteína C (4).

La enfermedad hepática es considerada en algunos estudios como el factor predisponente más importante para el diagnóstico de pileflebitis. Más del 50\% de los casos se asocian a enfermedad hepática severa.

La clínica usual de la pileflebitis inicia con la persistencia de un síndrome de respuesta inflamatoria sistémica luego del diagnóstico de infección intraabdominal a pesar del tratamiento antibiótico continuo y el manejo quirúrgico (2).

Las manifestaciones clínicas son inespecíficas pero la mayoría de pacientes se presentan con dolor abdominal, fiebre e incluso ictericia y puede llegar a manifestarse de forma más severa con shock séptico e insuficiencia hepática (5).

Los hallazgos más comunes en laboratorios corresponden a leucocitosis, anemia y alteración en pruebas de función hepática.

Para su diagnóstico son imprescindibles los estudios de imagen, como la ecografía abdominal, que demostrará la presencia de material ecogénico en el interior de la luz de los vasos o la tomografía computada que es el método de elección, la cual permite identificar los distintos focos infecciosos intraabdominales. Además la ecografía doppler puede proporcionar información sobre la alteración del flujo portomesentérico (5).

El proceso infeccioso usualmente es polimicrobiano, siendo E. coli, S. viridans y B. fragilis los organismos más comúnmente implicados. Además se han descrito estos dos agentes como los detectados con mayor frecuencia en pacientes con apendicitis.

La alta incidencia de bacteriemia por bacteroides en pacientes con pileflebitis sugiere que la naturaleza trombogénica del microorganismo juega un papel importante en la patogénesis de la tromboflebitis séptica. Las heparinasas producidas en la pared celular de los bacteroides son responsables del desarrollo de trombosis localizadas (6).

El pilar del tratamiento consiste en el uso de antibióticos de amplio espectro dirigidos contra bacilos gram- 


\section{CIENCIA\&SALUD}

negativos, anaerobios y estreptococos durante 4 semanas. Los pacientes con abscesos hepáticos de $<3$ $\mathrm{cm}$ deben recibir al menos seis semanas de tratamiento antibiótico. Los abscesos de $>3 \mathrm{~cm}$ usualmente requieren drenaje.

La laparotomía con trombectomía y el drenaje percutáneo también han sido descritos en los casos refractarios a tratamiento con antibióticos (7).

El papel de la anticoagulación en el tratamiento de la pileflebitis ha sido un tema controvertido. La evidencia sobre su uso se limita a reportes de casos y no se cuenta con estudios grandes con un diseño que permita establecer la significancia estadística a favor o en contra de su uso.

En el estudio de Plemmons, et. al. se observó una tasa de supervivencia de $100 \%$ entre los pacientes que recibieron anticoagulación con heparina en comparación con el $60 \%$ de los que no recibieron anticoagulación, sin embargo, estos resultados no fueron estadísticamente significativos (8).

Kanellopoulou, et. al. señalaron que el uso temprano de la anticoagulación en la trombosis de la vena porta puede minimizar las secuelas graves y acelerar la recanalización.

Choudhry, et. al. determinaron una tasa de mortalidad más baja que la reportada en la literatura, la cual puede atribuirse al uso de anticoagulación (9).

No existe un consenso sobre la duración del uso de la terapia de anticoagulación; ciertos estudios sugieren de 3 a 6 meses, siendo esta durante más tiempo o de forma indefinida en pacientes con estados de hipercoagulabilidad (9).

En general se recomienda la anticoagulación en las siguientes situaciones: trombosis portal aguda y extensa, progresión documentada de la trombosis desde el momento del diagnóstico, tras resección intestinal por isquemia secundaria a trombosis portal, fiebre persistente que no responde a antibióticos de amplio espectro o intervención quirúrgica y en ambos casos si se acompaña de pileflebitis supurativa y en pacientes con alteraciones de coagulación o estados de hipercoagulabilidad como en neoplasias y enfermedades hematológicas.

Las complicaciones detectadas con mayor frecuencia son los abscesos hepáticos múltiples (generalmente en el lado derecho debido al flujo sanguíneo de la vena mesentérica superior hacia el lóbulo hepático derecho) y la progresión de la trombosis hacia el sistema venoso entérico, lo que resulta en isquemia mesentérica. Otra posible complicación asociada son los daños secundarios a hipertensión portal como esplenomegalia, várices esofágicas y cavernomatosis portal.

A pesar de que la mortalidad ha disminuido significativamente con la introducción de antibióticos de amplio espectro, esta entidad continua teniendo una tasa de mortalidad poco despreciable que varía en la literatura, entre 19 y $32 \%$.

\section{CONCLUSIONES}

La pileflebitis sigue siendo una patología poco frecuente que se asocia infecciones intraabdominales, siendo actualmente la diverticulitis y la apendicitis las más comunes. Presenta una alta morbilidad y mortalidad temprana; sin embargo, el tratamiento prolongado con antibióticos y la anticoagulación se asocian a una baja tasa de recurrencia y una alta probabilidad de reperfusión del sistema portal. 


\section{REFERENCIAS BIBLIOGRÁFICAS}

1. Bolt RJ. Diseases of the hepatic blood vessels. In: Bockus HL, ed. Gastroenterolog)r. 4th ed. Philadelphia: WB Saunders, 1985: 3259-3277.

2. Belhassen-García M, Gomez-Munuera M, Pardo-Lledias J, Velasco-Tirado V, Perez-Persona E, Galindo-Perez I et al. Pylephlebitis: Incidence and prognosis in a tertiary hospital. Enfermedades Infecciosas y Microbiología Clínica. 2014;32(6):350-354.

3. Kanellopoulou T, Alexopoulou A, Theodossiades G, Koskinas J,et al. Pylephlebitis: an overview of non-cirrhotic cases and factors related to outcome. Scand J Infect Dis. 2010;42:804-811.

4. Baril N, Wren S, Radin R, Ralls P, Stain S. The role of anticoagulation in pylephlebitis. The American Journal of Surgery. 1996;172(5):449-453.

5. Pérez-Bru S, Nofuentes-Riera C, García-Marín A, Luri-Prieto P, Morales-Calderón M, García-García S. Pileflebitis: una extraña pero posible complicación de las infecciones intraabdominales. Cirugía y Cirujanos. 2015;83(6):501-505.

6. Liappis AP, Roberts AD, Schwartz AM, Simon GL. Thrombosis and infection: a case of transient anti-cardiolipin antibody associated with pylephlebitis. Am J Med Sci 2003; 325: 365-8.

7. Rea J, Jundt J, Jamison R. Pylephlebitis: Keep it in your differential diagnosis. The American Journal of Surgery. 2010;200(6):e69-e71.

8. Plemmons RM, Dooley DP, Longfield RN. Septic Thrombophlebitis of the Portal Vein (Pylephlebitis): Diagnosis and Management in the Modern Era. Clinical Infectious Diseases. 1995; 21(5):1114-1120.

9. Choudhry A, Baghdadi Y, Amr M, Alzghari M, Jenkins D, Zielinski M. Pylephlebitis: a Review of 95 Cases. Journal of Gastrointestinal Surgery. 2015;20(3):656-661.

10. Baddley JW, Singh D, Correa P, Persich NJ. Crohn's disease presenting as septic thrombophlebitis of the portal vein (pylephlebitis): Case report and review of the literature. Am J Gastroenterol 1999; 94: 847-9 